EESTI NSV TEADUSTE AKADEEMIA TOIMETISED. X KÖDE

FOOSIKALTS-MATEMAATILISTE JA TEHNILISTE TEADUSTE SEERIA. 1961, NR. 4

ИЗВЕСТИЯ АКАДЕМИИ НАУК ЭСТОНСКОН ССР. ТОМ Х

СЕРИЯ ФИЗИКО-МАТЕМАТНЧЕСКИХ И ТЕХНИЧЕСКИХ НАУК. 1961. N. 4

\title{
НОВАЯ РАДИАЦИОННАЯ НОМОГРАММА
}

\section{ХЕЛЬГИ НИИЛИСК}

В настоящее время широко известны графические методы подсчега потоков теп лового излучения атмосферы с помощью так называемых радиационных номограмм. Нанболее широкое распространенне получили номограммы Ф. Брукса ['], А. А. Дмитрие ва $\left[{ }^{2}\right]$, Р. Мюrге и Ф. Меллера $\left[^{[3,}{ }^{4}\right]$, Г. Робннсона $\left[{ }^{5}, 6\right]$, Ф. Н. Шехтер $\left[{ }^{7}, 8\right]$, В. Эльзассеpa $\left[^{9}\right]$ и Г. Ямамото $\left.{ }^{10}\right]$. K сожалению, все эти номограммы имеют некоторые недостат ки $\left[^{11-14}\right]$, главными из которых являются использование недостаточно надежных характеристик поглощения длинноволновой радиации атмосфёрными газами и рассмотрение ннтегральной функции в зависимости только от эффективного содержания водяного пара. Влиянне углекислого газа на поглощение учитывается при этом весьма приближенно. Поглощение теплового излучения атмосферным озоном названные номограммы совсем не принимают во внимание.

$\mathrm{y}_{\text {становлено }}\left[{ }^{13}, 14\right]$, что одним из главнейших факторов, определяющих значення потоков излучения, рассчитанных по той или иной номограмме, является функция пропускания. Поэтому для уточнения радиацнонных номограмм прежде всего необходимо пзучить имеющиеся колнчестенные харақтеристики поглощения длинноволновой радиации в атмосфере и на этой основе получить нанболее надежную функцию пропускания.

Исходя из вышеизложенного, целью настоящей работы было построение интегральной функции пропускания атмосферы с учетом совместного влияния водяного пара, углекислого газа и озона "* на поглощение теплового излучения в атмосфере. На основе этой новой функции пропускания решается задача построения радиационной номограммы, достаточно точной и удобной с практической точки зрения. Эта работа за. вершает серию исследований автора $\left[{ }^{14-18}\right]$, посвященных проблеме расчета потоков теплового излучення в атмосфере.

Количественные данные о поглощении длинноволновой радиации, использованные в настоящей работе для определения интегральной функции пропускания, приведены большей частью для температуры $300^{\circ} \mathrm{K}$, а также для обычных комнатных температур порядка 285 $295^{\circ} \mathrm{K}$. Поскольку при наземных температурах интегральная функция пропускания слабо зависит от температуры $\left[{ }^{10}, 13\right]$, полученную на основе упомянутых данных функцию пропускания можно считать достаточно надежной для промежутка температур $270-310^{\circ} \mathrm{K}$. Следует отметить, что интегральная функция пропускания рассчитывалась не для всего спектра $(0-\infty \mu)$, а для области $2,27-250 \mu$ (в участках спектра $0-2,27$ и $250-\infty$ к количества энергии теплового излучения ничтожны и, следовательно, практически не влияют на значение функции пропускания при атмосферных температурах).

- Только в номограмме Г. Ямамото принципнально правильно учитывается совместное в.тияние $\mathrm{H}_{2} \mathrm{O}$ и $\mathrm{CO}_{2}$ на поглощение теплового излучения в атмосфере.

$\because$ Қак нзвестно $\left[{ }^{13}\right]$, остальные атмосферные газы нграют незначительную роль в поглощении длннноволновой радиащни атмосферой.

5 ENSV TA Toimetised T-4 61 
Определению количественных характеристик поглощения радиацин водяным паром посвящено большое число работ $[1,12,13,20-42]$, результаты которых получены либо путем теоретических расчетов, либо на основе эксперимента (в естественных или лабораторных условиях). Отметим, что почти для всех областей спектра данные различных авторов существенно расходятся. В настоящей работе для определения интегральной функции пропускания по возможности использовались результаты нанболее новых и совершенных работ.

Значения поглощения радиации водяным паром в близкой инфракрасной области (интервалы спектра 2,27-2,99, 2,99-3,57 и 4,888,7 м) рассчитаны на основе соответствующих формул Д. Говарда, Д. Берга и Д. Вильямса [20]. Для участка $3,57-4,88 \mu$ экспериментальные данные отсутствуют. Известно только, что в этой области спектра нет полос поглошения водяным паром и поглощение обусловлено перекрыванием этого интервала крыльями полос поглощения, расположенными в соседних областях спектра. Можно полагать, что логарифмический коэффициент поглошения $k_{w}$ не превышает здесь $0,5 \mathrm{~cm}^{2} /$ г. Расчеты показали, что изменение коэффициента поглощения в пределах $0-0,5 \mathrm{~cm}^{2} / \mathrm{r}$ почти не влияет на значения функции пропускания 2,27 $8,7 \mu$ и тем более на значения интегральной функции пропускания. Исходя из этого, в настоящей работе функция пропускания $P$ для промежутка $3,57-4,88 \mu$ рассчитывалась по формуле

$$
P=e^{-k w^{w *}}
$$

нспользуя некоторое среднее значение $k_{w}=0.2 \mathrm{~cm}^{2} / \mathrm{r}$. (Здесь $w^{*}-$ эффективное содержание водяного пара.)

Для определения функции пропускания в области спектра $8,7-12$ ॥ нет достаточных оснований предпочитать результаты одного или другого автора (работы $[12,13,21,22,26,28,35,37-40])$. Поэтому в настоящеін работе функция пропускания для упомянутого интервала спектра рассцитывалась по формуле (1), используя некоторые средние значения коэффнциентов прглощения водяным паром (табл. 1).

Отметим, что, согласно новейшим Таблица 1 данным $\left[{ }^{37-39}\right], k_{w}$ около 9,6 и $11,1 \mu$ составляет примерно 0,1 .

В промежутке $9,0-10,3 \mu$ необхо.

Коэффициенты поглощения водяного пара для области спектра $8,7-12 \mu$

\begin{tabular}{c|c}
\hline $\begin{array}{c}\text { Область } \\
\text { спектра, } \mu\end{array}$ & $k_{w}, \mathrm{~cm}^{2} / \mathrm{r}$ \\
\hline $8,7-9,0$ & 0,15 \\
$9,0-11,5$ & 0,10 \\
$11,5-12,0$ & 0,20
\end{tabular}
димо учитывать влияние сильной полосы поглощения озона. Функция пропускания для участка $9,0-10,3$ !: найдена нами ранее [16].

В области $12-18 \mu$ газами, поглощающими тепловое излучение атмосферы, являются водяной пар, а также углекислый газ, так как в этом интервале находится сильная полоса поглощения углекислого газа. В настоящей работе использована функция пропускания для области $12-18 \mu$, полученная в работах $[15,18]$ на основе данных $\left[{ }^{19}\right]$ и $\left[{ }^{22}\right]$.

В работах $[13,21,22,27-30,36,41,42]$ приведены данные о поглощении длннноволновой радиации водяным паром в далекой инфракрасной области спектра. Сравнение результатов этих работ показывает, что

- Согласно $\left[{ }^{13}\right]$ влиянне остальных полос поглощения углекислым газом на перенос длинноволнового излучення в атмосфере можно не учитывать. 
с новейшими данными лабораторных измерений К. Палмера [36] наиболее близко совпадают результаты теоретических расчетов Г. Ямамото [22]. Исходя из этого, в настоящей работе функция пропускания для ннтервала $18-250 \mu$ определена на основе использования обобщенных коэффициентое поглощения водяным паром по данным работы $\left[{ }^{22}\right]$.

Отметим, что для всех рассмотренных интервалов спектра функция пропускания для диффузного излучения определялась по следующему нзвестному соотношению [13]:

$$
P_{F}\left(w^{*}\right)=2 \int_{0}^{\frac{\pi}{2}} P\left(w^{*} \sec \theta\right) \sin \theta \cos \theta d \theta,
$$

где $P_{F}-$ функция пропускания для диффузного излучения, $P-$ функция пропускания для направленного излучения, $\vartheta-$ зенитный угол.

На основе использования результатов определения функций пропускания для различных участков спектра рассчитана интегральная функция пропускання для диффузного излучения по следующей формуле:

$$
P_{F}=\frac{1}{f} \sum_{\Delta} f_{\Delta} P_{F\lrcorner}
$$

Здесь индекс $\Delta$ обозначает рассмотренные интервалы спектра, $f-$ доля интегрального нзлучения абсолютно черного тела, приходящегося на участок спектра $2,27-250 \mu, f_{\Delta}-$ доля излучения абсолютно черного тела, приходящаяся на интервал спектра $\Delta$.

Результаты расчетов приведены в таблицах 2 и 3. Здесь даны значения функций $\Delta P_{1}\left(w^{*}, u^{*}\right)$ и $\Delta P_{2}\left(w^{*}, m^{*}\right)$, с помошью которых искомая ннтегральная функция пропускания может быть вычислена по соотношению

$$
P_{F}\left(w^{*}, u^{*}, m^{*}\right)=0,001\left(\Delta P_{1}+\Delta P_{2}\right),
$$

где $u^{*}$ - эффективное содержание углекислого газа, $m^{*}$ - эффективное содержание озона.

Так как при вычислении таблиц 2 и 3 использованы также приближенные методы (численное интегрирование, интерполирование), то полученную функцию пропускания можно считать надежной с точностью до одной сотой.

Имея данные аэрологического зондирования, можно легко определить значения потоков теплового излучения в атмосфере, используя таблишы 2 и 3 и обычный графический метод, основанный на использовании соотношений (см. $\left.\left[{ }^{13}\right]\right)$

$$
G=\int P_{F} d B
$$

где $G$ - поток теплового излучения атмосферы, $B=\sigma T^{4}-$ интегральный поток излучения абсолютно черного тела.

Иными словами, $G$ численно равен площади в координатной системe $\left(P_{F}, B\right)$.

Номограмма для определения $G$ изображена на фиг. 1.

Следует отметить, что формула (5) выведена в предположении не-

" Значения $w^{*}, u^{*}$ н $m^{*}$ выражены в «см» (толщнна слоя осажденного вещества Б сантнметрах прн нормальных давленни и температуре). 


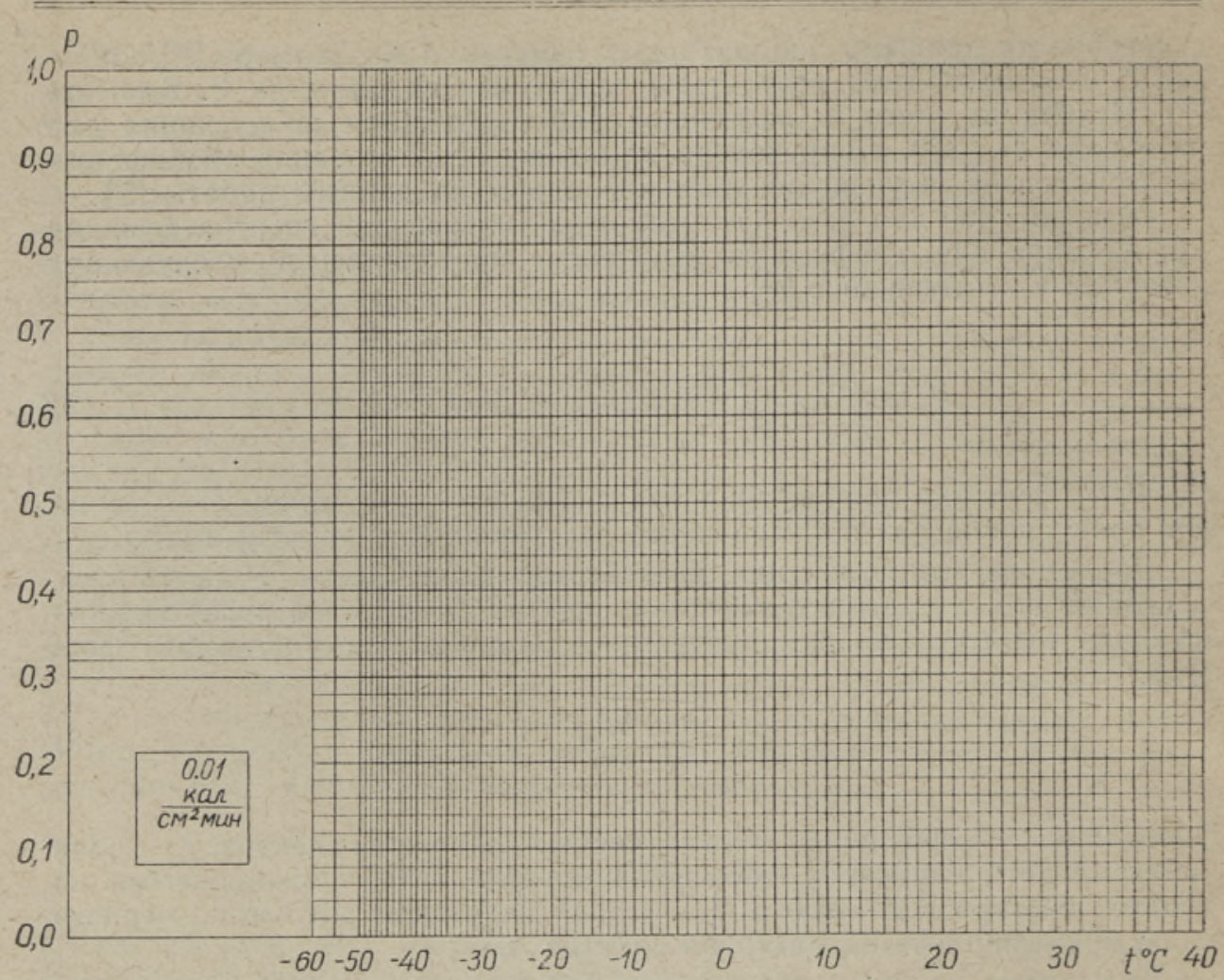

Фиг. 1. Номограмма для определения потоков длинноволновой раднации в атмосфере.

зависимости интегральной функции пропускания атмосферы от температуры $\left[{ }^{13}\right]$. Но, как известно $[2,4,9,10,13]$, в действительности функция пропускания зависит не только от содержания поглощающих веществ в атмосфере, но и от давления и температуры. Зависимость $P_{F}$ от давления учнтывается обычно при помощи эффективной поглощающей массы (см. $[1-10,13,14])$. Однако проблема учета температурной зависимости до сих пор окончательно не решена, и этот вопрос можно в некоторой степени считать дискуссионным [13].

В настояшей работе сделана попытка оценить влияние зависимости $P_{F}=P_{F}(T)$ на определение потоков теплового излучения атмосферы при помощи радиационной номограммы.

Решая общие уравнения переноса длинноволнового излучения в атмосфере, имеем следующее выражение для интенсивности нисходящей монохроматической радиации, распространяющейся в направлении $\vartheta\left[{ }^{13}\right]$ :

$$
I \lambda \downarrow(z, \vartheta)=\int_{z}^{\infty} \frac{k_{\lambda}(T, p)}{\cos \eta} \mathrm{Q}(\eta) E_{\lambda}(T) e^{-\frac{1}{\cos \vartheta} \int_{z}^{\eta} k_{\lambda}(T, p) \rho(\xi) d \xi} d \eta .
$$

Здесь $I \lambda \downarrow(z, \vartheta)$ - интенсивность нисходящей длинноволновой монохроматической радиации на уровне $z, k_{\lambda}(T, p)$ - коэффициент поглощения для длины волны $\lambda, \quad T=T(\eta)$ - температура поглощающей (излучающей) среды, $p=p(\eta)$ - общее давление в атмосфере, $\mathrm{Q}$ плотность вещества, поглощающего излучение, $E_{\lambda}(T)$ - интенсивность излучения абсолютно черного тела для длины волны $\lambda$, $\vartheta-$ зенитный угол. 


\begin{tabular}{|c|c|c|c|c|c|c|c|c|c|c|c|c|c|c|c|c|c|c|c|c|c|c|c|c|c|c|c|c|c|c|c|c|}
\hline & 00 & $\overline{1}, 00$ & $\overline{1}, 20$ & $\overline{1}, 40$ & $\overline{1}, 60$ & 80 & 0,00 & 0,10 & 0,20 & 0,30 & 0,40 & 0,50 & 0,60 & 0,70 & 0,80 & 0,90 & 1,00 & 1,10 & 1,20 & 1,30 & 1,40 & 1,50 & 1,60 & 1,70 & 1,80 & 1,90 & 2,00 & 2,10 & 2,20 & 2,30 & 2,40 & 2,50 \\
\hline$\overline{4}, 00$ & & 854 & 848 & 841 & 832 & 823 & 812 & 806 & 800 & 793 & 787 & 781 & 774 & 768 & 761 & 755 & 749 & 744 & 739 & 733 & 727 & 722 & 717 & 711 & 706 & 701 & 696 & 691 & 686 & 682 & 7 & s. \\
\hline$\overline{4,10}$ & 34 & 850 & 844 & 837 & 828 & 819 & 808 & 2 & 796 & 789 & 783 & 777 & 70 & 764 & 57 & 751 & 745 & 40 & 35 & 29 & 723 & 718 & 713 & 07 & 702 & 697 & 692 & 89 & 32 & 88 & 3 & \\
\hline 4,20 & 858 & 844 & 838 & 831 & 822 & 813 & 02 & & 790 & 783 & 777 & 771 & & & & 745 & & 734 & & 23 & 17 & & 707 & 11 & 96 & 691 & 86 & 81 & 66 & 72 & 37 & 33 \\
\hline$\overline{4,30}$ & 853 & 838 & 833 & 825 & 816 & 807 & 796 & & 784 & 77 & 771 & 765 & & & 15 & 739 & & 728 & & & 11 & 706 & 701 & 95 & 690 & 685 & 80 & 675 & 70 & 36 & 31 & 57 \\
\hline$\overline{4}, 40$ & 845 & 831 & 825 & 818 & 809 & 800 & 789 & & 777 & 7 & & 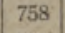 & & & & 732 & 726 & 721 & & & 4 & 99 & 694 & 8 & 33 & 678 & 73 & 668 & 33 & 59 & 54 & 50 \\
\hline$\overline{4}, 50$ & 838 & 823 & 817 & 810 & 801 & 792 & 781 & & 7 & 7 & & & & & & 724 & 718 & 1 & & & & 1 & 686 & & 5 & 670 & 65 & 660 & 55 & 51 & 56 & 12 \\
\hline$\overline{4}, 60$ & 8 & 815 & 809 & 802 & 793 & 784 & 773 & & & 7 & 748 & 742 & & 729 & 722 & 716 & 710 & 1 & & & & 83 & 67 & & 7 & 662 & 657 & 652 & 47 & 43 & 38 & 34 \\
\hline$\overline{4}, 70$ & 820 & 806 & 800 & 794 & 784 & 775 & 764 & 758 & & 7 & 739 & 733 & 120 & 720 & 713 & 707 & 701 & & & & & 74 & 9 & & 658 & 653 & 648 & 643 & 38 & 34 & 29 & 25 \\
\hline$\overline{4}, 80$ & 81 & 797 & 791 & 784 & 775 & 766 & 755 & 749 & & & 730 & 724 & 717 & 711 & 704 & 698 & 692 & & & 62 & 0 & 55 & 660 & 54 & 49 & 644 & 639 & 334 & 29 & 25 & 20 & e \\
\hline$\overline{4}, 90$ & 800 & 786 & 780 & 773 & 764 & 755 & 744 & 738 & 73 & 725 & 719 & 713 & 706 & 700 & 693 & 687 & 681 & 676 & 11 & 665 & 659 & 654 & 649 & 643 & 638 & 633 & 628 & 623 & 618 & 614 & 09 & 05 \\
\hline$\overline{3}, 00$ & 36 & 773 & 767 & 760 & 751 & 742 & 731 & 725 & $\begin{array}{l}719 \\
\end{array}$ & 712 & 706 & 700 & 693 & 687 & 680 & 674 & 668 & 663 & 658 & 652 & 646 & 641 & 636 & 630 & 625 & 620 & 615 & 510 & 305 & 01 & 96 & 592 \\
\hline 3,10 & 74 & 760 & 754 & 747 & 738 & 729 & 718 & 712 & 7 & 699 & 6 & 687 & 6 & 6 & 6 & 661 & & 65 & 5 & & 10 & 628 & & & & & 602 & 97 & & 8 & 3 & 79 \\
\hline$\overline{3}, 20$ & 759 & 746 & 740 & 733 & 724 & 715 & 704 & 698 & 692 & 6 & 679 & 673 & 6 & 660 & 6 & 647 & 6 & 63 & & 6 & 6 & 61 & & & & & 588 & 83 & 8 & 4 & 69 & 35 \\
\hline 3.30 & 743 & 730 & 724 & 716 & 707 & 698 & 687 & 681 & 675 & 66 & 6 & 6 & 6 & 643 & 6 & 6 & 10 & 619 & 6 & 608 & 6 & & 5 & & & & 572 & 67 & 2 & 88 & 53 & 49 \\
\hline$\overline{3}, 40$ & 728 & 714 & 708 & 701 & 692 & 683 & 672 & 606 & 600 & 6 & 6 & 6 & 634 & 628 & 621 & 615 & 609 & 6 & & 10 & 5 & 582 & 5 & & 566 & & 657 & 552 & & 13 & 38 & 34 \\
\hline$\overline{3}, 50$ & 714 & 700 & 694 & 687 & 678 & 669 & 658 & 652 & 646 & 6 & 6 & 627 & 620 & 614 & 607 & 601 & 595 & 5 & 585 & $1=$ & 5 & 568 & 5 & & 2 & 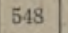 & 543 & 38 & & 29 & 24 & 20 \\
\hline$\overline{3}, 60$ & 700 & 686 & 680 & 673 & 664 & 655 & 644 & 639 & 633 & 626 & 619 & 613 & 606 & 600 & 593 & 588 & 582 & 57 & 572 & 5 & 5 & 55 & -1 & & 639 & 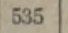 & 530 & 25 & 20 & 516 & 11 & 507 \\
\hline$\overline{3}, 70$ & 687 & 673 & 667 & 660 & 651 & 642 & 631 & 626 & 620 & 613 & 606 & 600 & 593 & 587 & 580 & 575 & 569 & 564 & 559 & 10 & 5 & 542 & 5 & 5 & 526 & 52 & 517 & 512 & 97 & 03 & 88 & 494 \\
\hline$\overline{3}, 80$ & 674 & 660 & 654 & 647 & 638 & 629 & 619 & 613 & 607 & 600 & 594 & 587 & 580 & 574 & 568 & 562 & 556 & 551 & 546 & 40 & 534 & 52 & 524 & 5 & 514 & 50 ? & 504 & 499 & 34 & 190 & 86 & 02 \\
\hline$\overline{3}, 90$ & 661 & 647 & 641 & 634 & 625 & 616 & 606 & 603 & 594 & 587 & 581 & 574 & 567 & 561 & 555 & 549 & 543 & 538 & 533 & 527 & 521 & 516 & 511 & 506 & 501 & 496 & 491 & 486 & 481 & 877 & 73 & 409 \\
\hline$\overline{2}, 00$ & 648 & 634 & 628 & 621 & 612 & 603 & 593 & 587 & 581 & 574 & 568 & 11 & 555 & 548 & 542 & 537 & 531 & 526 & 52 & 515 & 9 & 504 & 9 & 4 & 489 & 484 & 479 & 474 & 469 & 0 & 2 & 57 \\
\hline 2 & 634 & 620 & 614 & 607 & 599 & 590 & 580 & 574 & 568 & 561 & 5 & 548 & 542 & 535 & 5 & 523 & & & & & & & 4 & & & 471 & 466 & 61 & & 2 & 8 & 43 \\
\hline$\overline{2}, 20$ & 621 & 607 & 601 & 594 & 585 & 577 & 567 & 561 & 555 & 548 & & 53 & 28 & 522 & 5 & 510 & 5 & & & & & & & & & 458 & 153 & 148 & & 19 & 4 & 30 \\
\hline$\overline{2}, 30$ & 607 & 593 & 587 & 580 & 572 & 563 & 553 & 547 & 541 & 535 & 52 & 5 & 15 & 508 & 5 & 497 & & & & & & & & & & 444 & 39 & 134 & & 26 & 14 & 17 \\
\hline$\overline{2,40}$ & 592 & 578 & 572 & 566 & 558 & 548 & 538 & 533 & 527 & 520 & 51 & 50 & 00 & 494 & 48 & 483 & 4 & & & 4 & & & & & & 431 & 426 & 422 & & 12 & 88 & 04 \\
\hline 2,50 & 578 & 564 & 558 & 551 & 544 & 535 & 525 & 520 & 514 & 507 & 50 & 493 & 87 & 481 & 4 & 470 & & & 4 & 4 & & & 4 & & 23 & 41 & 413 & 108 & & 9 & 94 & 90 \\
\hline 60 & 562 & 549 & 543 & 537 & 529 & 520 & 510 & 505 & 498 & 492 & 485 & 47 & 73 & 467 & 46 & 456 & 45 & 445 & 44 & & 4 & 42 & 4 & 4 & 409 & 404 & 399 & 195 & & 37 & 32 & 78 \\
\hline 70 & 545 & 532 & 526 & 520 & 512 & 503 & 494 & 489 & 48 & 476 & 469 & 463 & 457 & 451 & 445 & 440 & 435 & 430 & 425 & 4 & 414 & 409 & 4 & 3 & 394 & 390 & 385 & 380 & 6 & 72 & 67 & 63 \\
\hline 86 & 529 & 516 & 510 & 504 & 496 & 487 & 478 & 473 & 46 & 461 & 454 & 44 & 442 & 436 & 430 & 425 & 420 & 415 & 0 & 404 & 399 & 395 & 390 & 38 & 38 & 376 & 371 & 367 & 362 & 58 & 354 & 350 \\
\hline 2,90 & 51 & 498 & 493 & 487 & 479 & 471 & 461 & 456 & -45 & 444 & 438 & 432 & 426 & 421 & 415 & 410 & 405. & 400 & 395 & 389 & 384 & 380 & 375 & 371 & 366 & 361 & 357 & 353 & 348 & 344 & 340 & 336 \\
\hline$\overline{1}, 00$ & 49 & 480 & 475 & 469 & 461 & 453 & 444 & 4 & 45 & 427 & 421 & 415 & 9 & 404 & 398 & 393 & 388 & 383 & 378 & 373 & 368 & 364 & 359 & 355 & 35 & 34 & 342 & 338 & 33 & 9 & 6 & 22 \\
\hline 1,10 & 47. & 460 & 454 & 448 & 441 & 433 & & 4 & & & 403 & 397 & 92 & 387 & 2 & 77 & 3 & & 2 & 3 & 352 & & 343 & 32 & 335 & 3. & 327 & 23 & 8 & & & 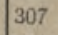 \\
\hline 1.20 & 45 & 440 & 435 & 429 & 422 & 414 & & & 396 & 390 & & 380 & & 9 & 5 & 360 & & & 5 & 3 & 3 & 33 & 327 & 3 & 320 & 316 & 312 & 308 & 4 & 0 & 7 & : \\
\hline 1,36 & $4:$ & 417 & 410 & 407 & 401 & 392 & 3 & 37 & 3 & 70 & 365 & 359 & & 349 & 5 & 340 & 3 & i0 & 326 & 322 & 3 & 3 & $3:$ & ? & 303 & 29 & 296 & 293 & 39 & 15 & ? & 12 \\
\hline 1,4 & 40 & 394 & 390 & 384 & 378 & 371 & 36 & 359 & 3 & 3 & 345 & 340 & 3 & 38 & 6 & 321 & 3 & 3 & 307 & 304 & 301 & 297 & 293 & 290 & 28 & 2 & 280 & 7 & 3 & 270 & t & 63 \\
\hline 1,50 & 3 & 369 & 365 & 360 & 354 & 347 & 34 & 338 & 3 & 3 & & 319 & 315 & 3 & 3 & 302 & 297 & 29 & 259 & 286 & 282 & 27 & 276 & 272 & 269 & 2 & 26 & 60 & 66 & 3 & 0 & 1 \\
\hline 1,60 & 35 & 341 & 338 & 332 & $32 ?$ & 320 & 31 & 311 & 3 & 3 & 301 & 297 & 293 & 289 & 285 & 281 & 278 & 274 & 270 & 2 & 264 & 26 & 258 & 256 & 25 & 250 & 2 & 14 & 240 & 237 & 1 & 12 \\
\hline 1,70 & 322 & 312 & 308 & 304 & 299 & 293 & 28 & 285 & 282 & 28 & 27 & 274 & 27 & 267 & 264 & 260 & 256 & 253 & 250 & 247 & 244 & 242 & 240 & 238 & 235 & 23 & 230 & 227 & 47 & 221 & 19 & 217 \\
\hline 1,80 & 292 & 283 & 280 & 277 & 274 & 269 & 25 & 251 & 258 & 25 & 25 & 250 & 247 & 24 & 242 & 239 & 236 & 233 & 230 & 227 & 225 & 224 & 222 & 220 & 218 & 21 & 212 & 209 & & 200 & 203 & 01 \\
\hline$\overline{1}, 90$ & 262 & 255 & 253 & 249 & 246 & 242 & 238 & 236 & 233 & 230 & 228 & 226 & 224 & 222 & 218 & 215 & 213 & 211 & 209 & 207 & 205 & 203 & 202 & 201 & 199 & 196 & 193 & 191 & 189 & 187 & 185 & 183 \\
\hline $0, C$ & & 22 & 22 & 221 & 2 & 21 & 2 & 2 & 1 & & 204 & 202 & 200 & 1 & 6 & 194 & 192 & 190 & 88 & 86 & & & 18 & & 177 & 176 & 174 & 173 & & 9 & 168 & 167 \\
\hline 0,1 & & 19 & 19 & 198 & 1 & 18 & 18 & 1 & 182 & 18 & 17 & 176 & 174 & 173 & 172 & 17 & 168 & 167 & 165 & 163 & 161 & 160 & 159 & 15 & 1 & 15 & & 4 & 152 & & 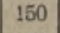 & 1 \\
\hline 0,2 & 17 & 167 & 166 & 16 & 162 & 16 & 1 & 1. & 15 & 15 & 15 & 151 & 150 & 14 & 148 & 147 & 146 & 145 & 144 & 143 & 142 & 14 & 140 & 13 & 15 & 13 & & 13 & & 3 & 2 & 1 \\
\hline 0,30 & & 13 & 13 & 13 & 13 & 13 & 1 & 1. & 131 & 13 & 12 & 128 & 127 & 127 & 126 & 126 & 125 & 125 & 124 & 123 & 122 & 121 & 120 & 119 & 1 & & & 11 & 180 & 115 & 114 & 1 \\
\hline 0,40 & 115 & 115 & 114 & 113 & 112 & 111 & 110 & 10 & 108 & 108 & 107 & 107 & 106 & 106 & 105 & 105 & 104 & 104 & 104 & 103 & 103 & 103 & 102 & 102 & 101 & 101 & 100 & 100 & 99 & 98 & 7 & 97 \\
\hline 0,50 & 9 & 9 & 9 & 9 & 9 & 8 & 8 & 8 & 8 & 88 & 87 & 8 & 8 & 8 & 86 & 86 & 8 & 85 & 84 & 84 & 84 & 84 & 83 & 83 & 83 & 8 & 82 & 8 & 2 & 81 & 1 & 81 \\
\hline 0,60 & 72 & 7 & 7 & 7 & 7 & 7 & 7 & 7 & 7 & 7 & 7 & 7 & 70 & 6 & 6 & 69 & 69 & 69 & 69 & 69 & 69 & 68 & 68 & 68 & 6 & 68 & 67 & 67 & . & 67 & 67 & 6 \\
\hline 0,7 & 56 & 5 & 5 & 5 & 5 & 5 & 5 & 5 & 5 & 5 & 5 & 5 & 5 & 5 & 55 & 55 & 55 & 55 & 55 & 55 & 54 & 54 & 54 & 54 & 54 & 34 & 53 & 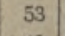 & & 53 & 3 & 53 \\
\hline 0,80 & 43 & 4 & 4 & 40 & 7 & 4. & 3 & a & 43 & 4 & 43 & 43 & 43 & 4 & 4 & 4 & 2 & 42 & 42 & 42 & 42 & 42 & 42 & 42 & 42 & 42 & 42 & 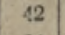 & 42 & 42 & 2 & 2 \\
\hline 0,90 & 32 & 32 & 32 & 32 & 32 & 32 & 32 & 32 & 32 & 32 & 32 & 32 & 32 & 32 & 3 & 31 & 31 & 31 & 31 & 31 & 31 & 31 & 31 & 31 & 31 & 31 & 31 & 31 & 31 & 31 & 31 & 31 \\
\hline & 23 & 23 & 23 & 23 & 23 & 23 & 23 & 23 & 23 & 23 & 23 & 20 & 23 & $23 \mid$ & $\pi$ & $\pi$ & 22 & 46 & 22 & 22 & 22 & 22 & 22 & 22 & $\because 2$ & 22 & 22 & 22 & 22 & 22 & 22 & 22 \\
\hline
\end{tabular}


зағ

pa:

прг

B $\dot{c}$

ле

мa

сил

Her

CTY

ры

MOI

MO]

ข

Зде

xpo

ще

(из

пло

изл

yro 
3 начения функиии $\Delta P_{1}\left(w^{*}, m^{*}\right)$

\begin{tabular}{|c|c|c|c|c|c|c|c|c|c|c|c|c|c|c|c|c|c|c|c|c|c|c|c|c|}
\hline - & & & & & & & & & & 0 & $\overrightarrow{2}, 70$ & 0 & $\overline{2}, 90$ & 00 & 10 & $|\overline{1}, 20|$ & $\overline{1}, 30$ & $\overline{1}, 40$ & $\overline{1}, 50$ & 60 & $\mid \overline{1}, 70$ & $\overline{1}, 80$ & & \\
\hline 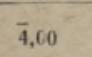 & 87 & 81 & 86 & 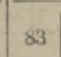 & 82 & 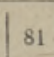 & 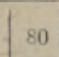 & 70 & 76 & 73 & 11 & , & 65 & 62 & 58 & 55 & 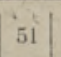 & 48 & 45 & 42 & 10 & 38 & 36 & \\
\hline$\overline{2}, 00$ & 87 & 87 & 86 & 83 & 82 & $8 !$ & 0 & 78 & 76 & 73 & 71 & 68 & 65 & 62 & 58 & 55 & 51 & 48 & 45 & 42 & 46 & 38 & 36 & 34 \\
\hline 1,00 & 86 & 85 & 84 & 81 & 80 & 79 & & 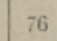 & 74 & 72 & 69 & $67^{\circ}$ & 64 & 61 & 57 & 53 & 0 & 47 & 44 & 41 & 39 & 37 & 5 & 2 \\
\hline & & 8 & 8 & & 79 & 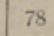 & & & 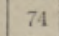 & 72 & 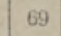 & & 61 & $6 c$ & 57 & 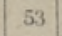 & 0 & & 44 & 41 & 39 & 7 & 5 & \\
\hline$i$, & 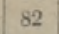 & 8 & 8 & & 78 & 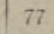 & & & 7 & 71 & . & . & 6 & 59 & 56 & 2 & 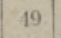 & 16 & 43 & 40 & 38 & 6 & 4 & \\
\hline $\mathrm{i}, \mathrm{f}$ & 80 & $8 x$ & of & 7 & 76 & 75. & 7 & 73 & 71 & 69 & 66 & 64 & 61 & 57 & 54 & 51 & 48 & 45 & 42 & 39 & 37 & 5 & 33 & \\
\hline T. & 77 & 77 & 7 & & 74 & 73 & 7 & 69 & 67 & 65 & 63 & 62 & 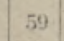 & 55 & 52 & 49 & 16 & 44 & 41 & 38 & 36 & 4 & 3 & \\
\hline & & & & & & 10 & & & & 65 & & & 3 & 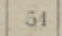 & Sit & 4s & & & & & & 1 & & \\
\hline & 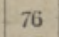 & & 7 & & 7 & 72 & & & 1 & 64 & 6 & & 5 & 54 & 51 & & & & & 7 & & 33 & +1 & \\
\hline$\div$ & 75 & & 7 & & 71 & 70 & & & 65 & 63 & 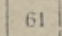 & 0 & 5 & 53 & 50 & 47 & . & 4 & 9 & 37 & & 33 & 1 & \\
\hline & 74 & 75 & $7:$ & 70 & 70 & 69 & 6 & 66 & 65 & 63 & 61 & 59 & 56 & 52 & 49 & 46 & 43 & 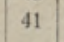 & 38 & 36 & 34 & 32 & 1 & 23 \\
\hline & 70 & 7 & 7 & 6 & 68 & 68 & G & & 64 & 62 & 60 & 58 & 5 & 51 & 48 & 45 & 43 & 40 & 38 & 35 & 4 & 2 & 80 & \\
\hline & 7 & & & & & os & & & 63 & .61 & 5 & 5 & & 50 & & 44 & & & & 1 & 33 & 31 & $\theta$ & is \\
\hline & 70 & & 6 & & & 6 & & & & 60 & & & & & & & & & & 34 & 3 & & & 7 \\
\hline & $6 x$ & & 6 & -6 & & 65 & & & & 59 & & & 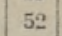 & 21. & & 42 & & & & 34 & & & & 7 \\
\hline & 67 & 6 & 64 & 6 & 61 & 63 & 62 & 61 & 60 & 58 & 55 & 54 & 51 & 47 & 44 & 41 & 39 & & 34 & 33 & 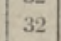 & (1) & 8 & 26 \\
\hline 0 . & 68 & 60 & 6 & & 63 & 62 & 6 & 60 & 50 & 57 & 55 & 53 & 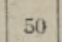 & 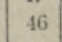 & 43 & 4) & $3 s$ & 36 & 34 & 32 & & & & 6 \\
\hline & 64 & 6 & 6 & & & 60 & & & & 3 & 5 & & & 80 & & & & 35 & 33 & 31 & & & & 25 \\
\hline & 62 & 6 & - & 5 & 5 & 58 & & & & 5 & 5 & 5 & & 44 & & & & & 2 & 1 & & 28 & 5 & A \\
\hline & . & 6 & 5 & 57 & 57 & 56 & 5 & & & 52 & 5 & & & 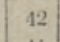 & & & & & 1 & 29 & 28 & $2 i$ & & 23 \\
\hline 0,36 & 58 & 5 & 5. & 56 & 56 & 55 & $s$ & 53 & 52 & 51 & $x$ & 48 & 45 & 41 & 39 & 36 & 34 & 32 & 30 & 28 & 27 & 25 & 5. & 23 \\
\hline 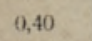 & 5 & 5 & 55 & & $=$ & 53 & 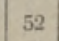 & 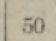 & 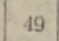 & 48 & 47 & & 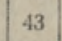 & 40 & 38 & 35 & 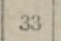 & 3 & 29 & 27 & & 25 & 21 & 22 \\
\hline & 5 & & & 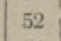 & 5 & 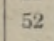 & & & & 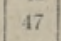 & & & & & 3 & 3 & & 30 & & 26 & & 24 & & 21 \\
\hline & 2 & 5 & 5 & $=$ & 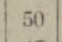 & 45 & & 4 & 4 & 4 & 4 & 42 & 39 & & & 3 & & & & 25 & & 23 & 2 & 0 \\
\hline & 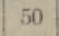 & 5 & 4 & 4 & 47 & 40 & 2 & 4 & 4 & 42 & 4 & 40 & & & & 3 & 29 & 20 & 26 & 24 & & 22 & 21 & 19 \\
\hline & 45 & 4 & 47 & 45 & 45 & 44 & 4: & 42 & 4 & 40 & 3 & 38 & 3 & 34 & 32 & 30 & 28 & 27 & 25 & 23 & 22 & 21 & 0 & 18 \\
\hline & 45 & 4 & 44 & 4 & 4: & 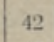 & & & & 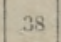 & 37 & & 34 & 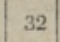 & 30 & 28 & 26 & $2:$ & 24 & 22 & & 20 & 19 & 17 \\
\hline & 4 & 4 & & & 4 & 4 & & & & 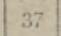 & & & & & & & 25 & 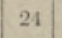 & 22 & 21 & & 19 & 8 & \\
\hline & 4 & 4 & 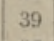 & & 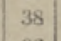 & 3 & & & & 3 & 3 & & 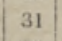 & s & & 26 & 21 & 23 & & 20 & & 18 & 17 & 5 \\
\hline & 3 & 3 & 3 & & $3 t$ & w & & & & 32 & 3 & & 29 & & 20 & 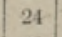 & 22 & 21 & - & 19 & & 17 & 16 & 1 \\
\hline 0,7 & 34 & 34 & 3 & 3 & 33 & $3:$ & 32 & 31 & 31 & 30 & 29 & 28 & $2 T$ & 25 & 24 & 23 & 21 & 20 & 18 & 17 & 17 & 16 & 5 & 13 \\
\hline & 31 & 31 & 3 & & 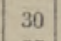 & 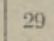 & & & & 27 & & & & 23 & 22 & 21 & 19 & 18 & & 15 & 15 & 14 & 13 & 2 \\
\hline & 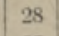 & 2 & & & 2 & 2 & & & & & & & & & 20 & & & 16 & & 14 & 13 & 3 & 2 & \\
\hline & 25 & 2 & 2 & 2 & . & 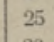 & & & & 23 & 2 & & & & 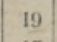 & 1 & 16 & 15 & & 13 & & 12 & $\pi$ & 10 \\
\hline & 23 & 2 & 2 & 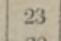 & 23 & 23 & 2 & 2 & 2 & 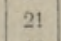 & 2 & & 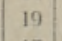 & . & 17 & 0 & 10 & 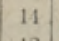 & 13 & 12 & 11 & 11 & 10 & 9 \\
\hline & 21 & 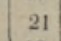 & 21 & 20 & 20 & 20 & I. & 19 & 19 & 18 & 18 & 17 & 17 & 16 & 15 & 14 & 13 & 12 & 11 & 11 & 10 & 10 & 9 & \\
\hline 1,00 & 19 & 19 & 19 & 18 & 18 & 18 & 17 & 17 & 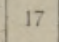 & 16 & 16 & & 15 & 14 & 13 & 12 & 11 & 10 & 10 & 9 & 9 & 8 & 8 & 7 \\
\hline
\end{tabular}

Выразим коэффициент поглощения следующим образом:

$$
k_{\lambda}(T, p)=k_{0 \lambda} F(T, p) \text {, }
$$

где $F(T, p)$ - некоторая функция от температуры и давления ( $k_{0}$ мы считаем независимым от $T$ и $p$ ).

С учетом (7) выражение (6) будет иметь следующий вид:

$$
I_{\lambda} \downarrow=\int_{z}^{\infty} \frac{k_{0 \lambda}}{\cos \vartheta} F(T, p) \varrho(\eta) E \lambda(T) e^{-\frac{k_{0} \lambda}{\cos \vartheta} \int_{z}^{\eta} F(T, p) \rho(\xi) d \Sigma} d \eta .
$$


Введем эффективную поглощающую массу $w^{*}$, которая олределяется следуюшим образом:

$$
w^{*}=\int_{z}^{y_{y}^{\gamma}} F(T, p) Q(\xi) d \xi .
$$

В таком случае

$$
I_{\lambda \downarrow} \downarrow=\int_{0}^{w_{\infty}^{*}} \frac{k_{0 \lambda}}{\cos \vartheta} E_{\lambda}(T) e^{-\frac{k_{0 \lambda}}{\cos \theta} w^{*}} d w^{*} .
$$

Интегрируя выражение (10) по $\lambda$ при помощи метода АмбарцумянаЛебединского [13], имеем:

$$
I \downarrow=\int_{0}^{w_{\infty}^{*}} E(T) d w^{*} \cdot \int_{0}^{\infty} \frac{k_{0}}{\cos \vartheta} l\left(k_{0}, T\right) e^{-\frac{k_{0}}{\cos \vartheta} w^{*}} d k_{0} .
$$

Здесь преднолагалось, что в участках спектра, для которых выполняется неравенство $\left.k_{0}<k_{0}\right\rangle<k_{0}+d k_{0}$, значение коэффициента поглощения можно практически считать постоянным $\left(k_{0}\right)$. Функция $f\left(k_{0}, T\right)$ определяет долю интенсивности абсолютно черного тела, приходящуюся на совокупность участков спектра, которым соответствует коэффи циент поглощения $k_{0} . E(T)$ в выражении (11) обозначает интегральную интенсивность излучения абсолютно черного тела.

С другой стороны, легко показать, что с использованием эффективной поглощающей массы, которая определена по формуле (9), интегральная функция пропускания атмосферы выражается следующим образом:

$$
P\left(w^{*}, T_{1}\right)=\int_{0}^{\infty} f\left(k_{0}, T_{1}\right) e^{-\frac{k_{0}}{\cos w^{*}}} d k_{0} .
$$

Здесь $T_{1}$ обозначает температуру источника излучения, причем предполагается, что этим источником является абсолютно черное тело.

Далее, введем новую функцию $P^{*}$, которая определяется соотношением

$$
P^{*}\left[w^{*}, T\left(w^{*}\right)\right]=P\left[w^{*}, T_{1}=T\left(w^{*}\right)\right] .
$$

Другими словами,

$$
P^{*}\left[w^{*}, T\left(w^{*}\right)\right]=\int_{0}^{\infty} f\left[k_{0}, T\left(w^{*}\right)\right] e^{-\frac{k_{0}}{\cos w^{*}}} d k_{0} .
$$

Найдем теперь производные $P$ и $P^{*}$ по $w^{*}$ :

$$
\frac{d P}{d w^{*}}=-\int_{0}^{\infty} \frac{k_{9}}{\cos \vartheta} f\left(k_{0}, T_{1}\right) e^{-\frac{k_{0}}{\cos \vartheta} w^{*}} d k_{0}
$$


Здесь предполагалось, что температура $T_{1}$ не изменяется.

$$
\begin{gathered}
\frac{d P^{*}}{d w^{*}}=-\int_{0}^{\infty} \frac{k_{0}}{\cos \eta} f\left(k_{0}, T\right) e^{-\frac{k_{0}}{\cos i t} w^{*}} d k_{0}+ \\
+\frac{d T}{d w^{*}} \int_{0}^{\infty} \frac{\partial f\left(k_{0}, T\right)}{\partial T} e^{-\frac{k_{0}}{\cos i} w^{*}} d k_{0} . \\
\text { Видим, что интеграл } \quad S_{1}=\int_{0}^{\infty} \frac{k_{0}}{\cos i} f\left(k_{0}, T\right) e^{-\frac{k_{0}}{\cos \vartheta} w^{*}} d k_{0} \quad \text { в выра- }
\end{gathered}
$$
жении (11) равен первому члену выражения (16). Следовательно, использование $\frac{d P^{*}}{d w^{*}}$, а также $\frac{d P}{d w^{*}}$ вместо $S_{1}$ в обоих случаях связано с погрешностями. В первом случае мы не учитываем второй член выражения (16), а во втором случае не учтен «эффект смещения».

Попытаемся приближенно оценить величины этих погрешностей. При средних условиях в тропосфере * связь между $T$ и * $^{*}$ хорошо описывается формулой

$$
T=-8\left(w^{*}\right)^{8}-10 w^{*}+20,
$$

где $w^{*}$ выражена в «см»и $T$ в ${ }^{\circ} \mathrm{C}$.

Для упрощения расчетов используем вместо выражений (12) и (14) приближенные формулы

$$
P\left(w^{*}, T_{1}\right)=\sum_{j=1}^{6} f_{j}\left(T_{1}\right) e^{-k_{j} w^{*}},
$$

где $T_{1}$ считается постоянным, и

где $T=T\left(w^{*}\right)$.

$$
P^{*}\left(w^{*}, T\right)=\sum_{j=1}^{6} f_{i}(T) e^{-k_{j} w^{*}},
$$

Формулы (18) и (19) взяты из монографии К. Я. Кондратьева [13].

В этой работе даны также значения коэффициентов поглощения $k_{i}$ и связь $f_{j}$ с температурой:

$$
f_{j}=a_{j}+b_{j} T .
$$

Здесь $a_{j}$ и $b_{j}$ - некоторые постоянные для каждого участка $j$.

Исходя из приведенных выше данных, в настоящей работе рассчитывались следующие величины: $S_{1}, S_{2}=\frac{d P^{*}}{d w^{*}}, S_{3}=\frac{d P\left(T_{1}=260^{\circ} \mathrm{K}\right)}{d w^{*}}$, $S_{4}=\frac{d P\left(T_{1}=300 \mathrm{~K}\right)}{d w^{*}}$. Результаты этих расчетов приведены на фиг. 2. Как видно из этого рисунка, использование $\frac{d P^{*}}{d w^{*}}$ вместо $S_{1}$ связано с большими погрешностями, особенно в верхних слоях трөпосферы. Разницы между $S_{1}$ и $S_{3}$, а также между $S_{1}$ и $S_{4}$ довольно малы. Поэтому следует

- Предполагается, что вертикальный градиент температуры равен 6 град/км, а плотность водяного пара $\varrho_{w}$ уменьшается с высотой по экспоненциальному закону. На уровне земной поверхности $T=20^{\circ} \mathrm{C}$ и $\mathrm{g}_{w}=7 \times 10^{-6} \mathrm{r} / \mathrm{cm}^{3}$. 
считать вполне обоснованным использование в формуле (11) $\frac{d P\left(w^{*}, T_{1}\right)}{d w^{*}}$ вместо $S_{1}$ (в предположении, что $T_{1}$ представляет собой не которую среднюю температуру в атмосфере). Другими словами, влиянием эффекта смещения в данном случае можно пренебречь.

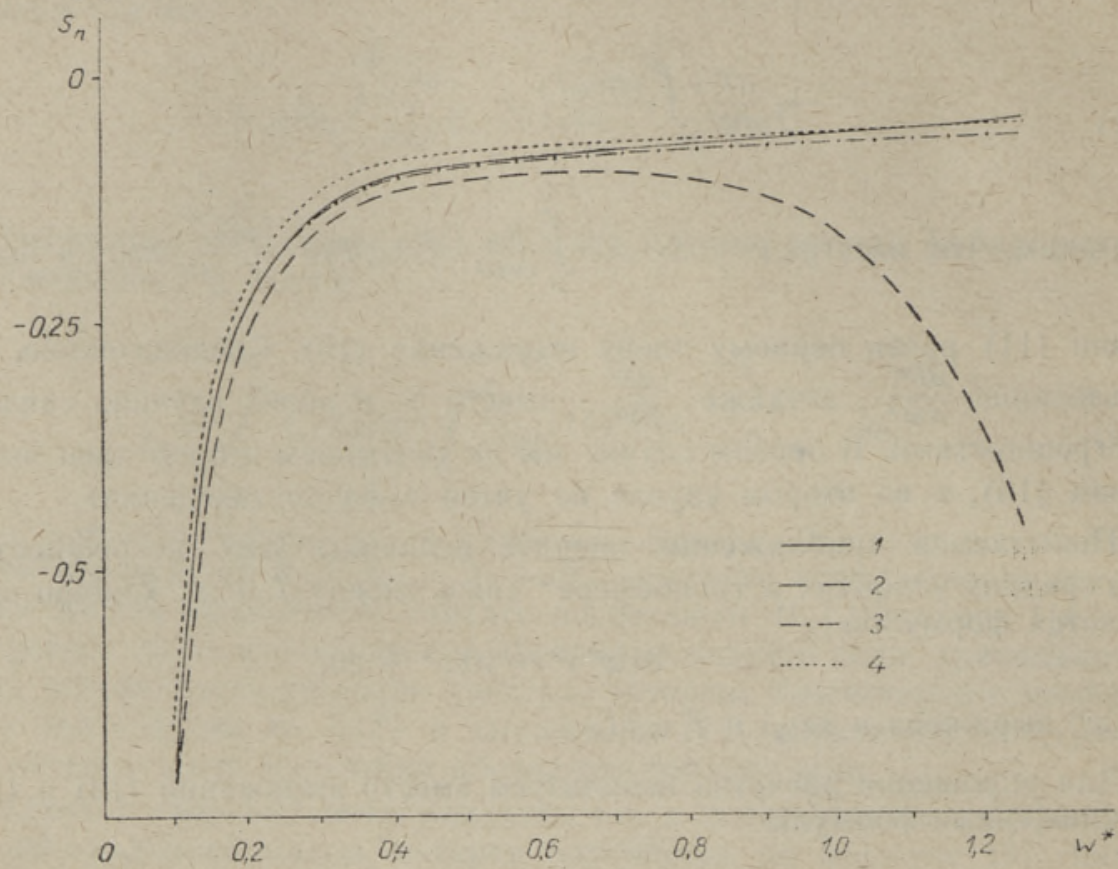

- Фиг. 2. Сравнение функций $S_{n}: 1-S_{1}, 2-S_{2}, 3-S_{3}, 4-S_{4}$.

Как известно $\left[{ }^{13}\right]$, зависимость коэффициента поглощения от температуры и «эффект смещения» действуют в противоположных направлениях. Поэтому следует считать оправданным и то, что не учитывается зависимость $k_{\lambda}=k_{\lambda}(T)$. В таком случае формула (9) имеет следующий вид:

$$
w^{*}=\int F(p) \varrho(z) d z,
$$

где $F(p)$ - некоторая функция от давления.

Исходя из вышеизложенного, вместо (11) получим

$$
I \downarrow=\int_{w_{\infty}^{*}}^{0} E(T) d w^{*} \frac{d P}{d w^{*}}=\int_{P\left(w_{\infty}^{*}\right)}^{1} E(T) d P .
$$

Интегрируя выражение (22) по частям, получаем выражение, аналогичное формуле (5):

$$
I \leftarrow=\oint P d E .
$$

Таким же образом можно получить выражение и для $I \uparrow$.

Отметим, что приведенные выше результаты оправданы и для потоков длинноволнового излучения в атмосфере. Как известно [13], потоки излучения рассчитываются путем интегрирования соответствующих интенсивностей по всем телесным углам, составляюшим полусферу. В таком случае вместо формулы (23) будем иметь формулу (5). 
Резюмируя приведенные выше результаты, можно сказать, что на основе приближенной формулы $G=\int P_{F}\left(w^{*}\right) d B$ можно с достаточной точностью определить длинноволновые потоки излученкя атмосферы, но нспользование формулы $G=\int P_{F}\left[w^{*}, T\left(w^{*}\right)\right] d B$ следует считать неоправданным.

Как было показано в работах $\left[{ }^{16}\right]$ и $\left[{ }^{43}\right]$, атмосферный озон играет лишь незначительную роль в определении значений интегральных потоков тепловой радиации в нижней тропосфере. Поэтому влиянием озона на поглошение длинноволнового излучения в атмосфере можно пренебречь при расчетах нисходящих потоков (G $\downarrow$ ) в нижней тропосфере и восходя!цих потоков $(G \uparrow)$ до $15-20$ км. В таком случае вместо таблиц 2 и 3 можно использовать лишь одну таблицу, в которой приведены значения интегральной функции пропускания $P_{F}\left(w^{*}, u^{*}\right)$.

В качестве примера нами рассчитаны нисходящий поток, восходяиций поток и эффективное излучение $(F=G \uparrow-G \downarrow)$ на уровнях $0 ; 3$ и 8 км для некоторых широтных зон земного шара. При этом использовались стратификации температуры, влажности и давления, взятые из работ [10, 44, 45]. Данные о вертикальном распределении озона получены из работы [46]. Концентрация углекислого газа в атмосфере принята равной $0,03 \%$ (по объему) для всех зон, так как о широтном ходе $\mathrm{CO}_{2}$ не было данных. При расчете эффективных поглощающих масс водяного пара мы применили обычно используемую поправку на давление $\left(p / p_{0}\right)^{0,5}$, а для углекислого газа и озона эта поправка была $\left(p / p_{0}\right)^{0.8}$ и $\left(p / p_{0}\right)^{0.2}$ соответственно (см. $\left.[15,16,18]\right)$. Результаты этих pacчетов приведены в табл. 4.

Таблица 4

Значения потоков теплового излучения в атмосфере (кал/см² мин)

\begin{tabular}{|c|c|c|c|c|c|c|}
\hline \multirow{2}{*}{$\begin{array}{l}\text { IІиротная } \\
\text { зона }\left({ }^{\circ} \mathrm{N}\right)\end{array}$} & \multicolumn{2}{|c|}{$s=0$} & \multicolumn{2}{|c|}{$z=3$} & \multicolumn{2}{|c|}{$z=8$} \\
\hline & $G$ & $F$ & $G$ & $F$ & $G$ & $F$ \\
\hline $0-10$ & 0,574 & 0,093 & 0,392 & 0,194 & 0,164 & 0,296 \\
\hline $10-20$ & 0,545 & 0,101 & 0,348 & 0,209 & 0,132 & 0,317 \\
\hline $20-30$ & 0,507 & 0,114 & 0,312 & 0,220 & 0,117 & 0,313 \\
\hline $30-40$ & 0,429 & 0,117 & 0,275 & 0,208 & 0,097 & 0,293 \\
\hline $40-50$ & 0,359 & 0,122 & 0,240 & 0,198 & 0,082 & 0,270 \\
\hline $50-60$ & 0,296 & 0,125 & 0,201 & 0,187 & 0,076 & 0,246 \\
\hline $60-70$ & 0,236 & 0,129 & 0,169 & 0,172 & 0,077 & 0,218 \\
\hline
\end{tabular}

В работах $[14,17]$ произведены аналогичные расчеты при помощи номограмм Ф. Брукса, А. А. Дмитриева, Р. Мюгге и Ф. Меллера, Г. Робинсона, Ф. Н. Шехтер, В. Эльзассера и Г. Ямамото. Сравнивая результаты определения $G \downarrow, G \uparrow$ и $F$, полученные в настоящей работе и в работах $\left[{ }^{14},{ }^{17}\right]$, видим, что вблизи земной поверхности и в нижней части тропосферы наши результаты довольно близко согласуются с данными, полученными по номограммам Ф. Шехтер и Ф. Брукса. Однако при $z=8$ км получается более близкое соответствие с результатами вычислений по номограммам Ф. Меллера и Г. Робинсона.

K сожалению, в настоящее время отсутствует достаточно полный комплекс экспериментальных данных, что не дает возможности провести прямое сравнение результатов с экспериментом.

Поскольку предложенная в настоящей работе радиационная номограмма построена в результате нанболее тщательного анализа современных данных о поглощенин инфракрасной ралиащии в атмосфере, можно считать, что она является наиболее надежной.

6 ENSV TA Toimetised T-4 61 


\section{Л ИТ Е Р А Т Р А}

1. F. A. Brooks, Atmospheric Radiation and Its Reflection from the Ground, Journ. Met., V. 9, No. 1, 1952.

2. А. А. Д м и т и ев, K вопросу о радиационном балансе атмосферы, Метеорол. и гидрология, № $11,1940$.

3. F. Mölle r, Grundlagen eines Diagramms zur Berechnung langwelliger Strahlungsströme, Meteor. Zeitschr., B. 61, H. 2., 1944.

4. F. Mölle r, Long-Wave Radiation, Compendium of Meteorology, 1951, pp. 34-49.

5. G. D. Robinson, Notes on the Measurement and Estimation of Atmospheric Radiation I, Quart, Journ. Roy. Met. Soc., V. 73, No. 315-316, 1947.

6. G. D. Robinson, Notes on the Measurement and Estimation of Atmospheric Radiation II, Quart. Journ. Roy. Met. Soc., V. 76, No. 327, 1950.

7. Ф. Н. Шехте р, К вычислению лучистых потоков тепла в атмосфере, Тр. ГіО, вып. $22(84), 1950$.

8. Ф. Н. Шехт ер, Расчет лучистых потоков длинноволновой радиации из ограниченного телесного угла и полупространства, Тр. ГГО, вып. 39 (101), 1953.

9. W. M. E Is a s se r, Heat Transfer by Infrared Radiation in the Atmosphere Harv, Met. St., No. 6, 1942

10. G. Y a m a moto, On a Radiation Chart, Sci. Rep. Tôhoku Univ., Ser. 5, V, 4, No. 1, 1952.

11. К. Я. Конд ратьев, Некоторые вопросы лучистого теплообмена в атмосфере, Уч. зап. ЛГУ, сер. физ., вып. 7, 1949.

12. К. Я. Конд ра тьев, Перенос длинноволнового излучения в атмосфере, М.-Л., Гостехиздат, 1950.

13. К. Я. Кондратьев, Лучистый теплообмен в атмосфере, М., Гидрометеонздат, 1956.

14. Х. Ю. Н и й л и ск, К вопросу о расчетах теплового излучения атмосферы, Исследования по физике атмосферы, ИФА АН ЭССР, № 2, 1960.

15. и 16. К. Я. К онд р а тьев, Х. Ю. Н и йли ск, К вопросу о тепловом излучении углекислого газа в атмосфере; О тепловом излучении $9,6 \mu$ полосы поглощения озона в атмосфере. Научн. сообщ. Ин-та геол. и геогр. АН Лит. ССР, т. 13 , вып. 2, 1962 (в печати).

17. K. Y. Kondratiev. H. J. Ni ilisk, Comparison of Radiation Charts, Geofisica pura e applicata, V. 46, 1960/II.

18. K. Y. Kondratiev, H. J. Niilisk, On the Question of Carbon Dioxide Heat Radiation in the Atmosphere, Geofisica pura e applicata, V. 46, 1960/II.

19. G. Y a m a moto, T. S a s a m or i, Calculation of the Absorption of the $15 \mu$ CarbonDioxide Band. Sci. Rep. Tôhoku Univ., Ser. 5, V. 10, No. 2, 1958.

20, J. N. H o w a r d, D. E. B u r c h, D. W i 11 i a m s, Near Infrared Transmission through Synthetic Atmospheres, Journ. Opt. Soc. Am., V 45, No. 3, 4, 5, 6, 1956.

21. W. E Is a s ser, New Values for the Infra-Red Absorption Coefficient of Atmospheric Water Vapour, Monthly Weath. Rev., V. 66, No. 6, 1938.

22. G. Yam amoto, G. On ishi, Absorption Coefficient of Water Vapour in the Far Infra-Red Region, Sci. Rep. Tôhoku Univ., Ser. 5, V. 1. No. 1, 1949.

23. G. Y a mamoto, G. O n ishi, Absorption Coefficient of Water Vapour in the Near Infra-Red Region, Sci. Rep. Tôhoku Univ., Ser. 5., V. 1, No. 2, 1949.

24. A. A d e 1, Atmospheric Absorption of Infrared Solar Radiation at the Lowell Observatory I, Astrophys. Journ., V. 89, No. 1, 1939.

25. A. A de l, C. D. La m p la nd, Atmospheric Absorption of Infrared Solar Radiation at the Lowell Observatory II. Astrophys. Journ., V. 91, No. 1, 1940.

26. A. Ade l, C. D. L a m pla n d, Atmospheric Absorption of Infrared Solar Radiation at the Lowell Observatory III, IV, Astrophys. Journ., V. 91, No. 5, 1940.

27. A. A de 1, An Estimate of Transparency of the Atmospheric Window $16 \mathrm{mu}$ to $24 \mathrm{mu}$, Journ. Opt. Soc. Am., V. 37, No. 10, 1947.

28. R. Anthony, Atmospheric Absorption of Solar Infrared Radiation, Phys. Rev., V. 85 , No. $4,1952$.

29. T. C. Cowling, The Absorption of Water Vapour in the Far Infra-Red, Rep. on Progr. in Phys., V. IX, 1943.

30. T. C. C ow l in g, Atmospheric Absorption of Heat Radiation by Water Vapour, Phil. Mag., V. 41, Ser. 7, No. 313, 1950.

31. T. Eld er, J. Strong, The Infra-Red-Transmission of Atmospheric Windows, Journ. Frankl. Inst., V. 255, No. 3, 1953.

32. F. E. F ow le, The Spectroscopic Determination of Aqueous Vapour, Astrophys. Journ., V. 35 , pp. $149-162,1912$.

33. F. E. Fow le, The Transparency of Aqueous Vapour, Astrophys. Journ., V. 42, pp. $394-411,1915$.

34. H. A. Gebbie, W. K. Harding, C. Hilsum, A. W. Price, V. Roberts, Atmospheric Transmission in the $1-14 \mu$ Region, Proc. Roy. Soc., V. 206, No. $1084,1951$. 
35. R. M. Goody, W. T. R o a ch, Absorption and Emission in the Atmospheric Window from $770-1250 \mathrm{~cm}^{-1}$, Quart. Journ. Roy. Met. Soc., V. 84, No. 362, 1958.

36. C. H. Palmer, Long Path Water Vapour Spectra with Pressure Broadening I, II, Journ. Opt. Soc. Am., V. 47, No. 11, 1958.

37. F. S a i e dy, Background (Continuum) Extinction in the Atmosphere, Oxford Radiation Symposium, Abstract No, 30, 1959.

38. F. S a i ed y, R. M. G o o dy, The Solar Emission Intensity at $11 \mu$, Monthly Not. Roy. Astr. Soc., V. 119, No. 3, 1959.

39. E. Vigroux, Emission Continue de l'atmosphère terrestre à $9.6 \mu$. Ann. Geophys., t. 15, № 4,1959 .

40. В. Г. К а с т о в, Измерение эффективного излучения в области максимальной прозрачности водяного пара, Метеорол. и гидрология, Инф. сб. №1, 1946.

41. В. Г. К астров, Измерения ннфракрасного излучения атмосферы в трех участках спектра, Тр. ЦАО, вып. 3, 1948.

42. Н. Г. Ярославский, А. Е. С таневич, Вращательный спектр $\mathrm{H}_{2} \mathrm{O}$ длннноволновой инфракрасной области 50-1500 $\mu$, Оптика н спектроскопия, т. V, вып. 4, 1958.

43. К. Я. Кондратьев, Т. Д. Матрешина, О влиянии длинноволнового нзлучения озона на раднационный баланс земной поверхности и атмосферы. Тр. ГГО, вып. 41 (103), 1953.

44. J. Lo n d o n, The Distribution of Radiational Temperature Change in the Northerm Hemisphere During March. Journ. Met., V. 9, No. 2, 1952.

45. G. O hring. The Radiation Budget of the Stratosphere, Journ. Met., V. 15, No. 5. 1958.

46. K. R. R a man at ha n, R. N. Kulkarni, Mean Meridional Distributions of Ozone in Different Seasons Calculated from UMKEHR Observations and Probable Vertical Transport Mechanisms, Quart. Journ. Roy. Met. Soc., V. 86, No. 368, 1960.

Ннститут физики и астрономии

Академии наук Эстонкой ССР

\section{Поступила в редакцию}

11. VI 1961

\title{
UUS KIIRGUSNOMOGRAMM
}

\author{
Helgi Niilisk
}

\section{Resümee}

Käesoleva töö eesmärgiks on täiustada atmosfääri inírapunaste kiirgusvoogude graafilisi arvutusmeetodeid. Kasutades eksperimentaalsete ja teoreetiliste uurimuste uusimaid andmeid on määratud atmosfääri integraalne läbilaskefunktsioon, mis arvestab veeauru. süsihappegaasi ja osooni mōju infrapunasele kiirgusele Maa atmosfääris. Saadud läbilaskefunktsiooni alusel on konstrueeritud uus, täiustatud kiirgusnomogramm. Selle abiI on arvutatud infrapunaste kiirgusvoogude keskmised väärtused maakera mitmesuguste vööndite jaoks $0 ; 3$ ja 8 km kōrgusel, kusjuures on arvestatud atmosfääri rōhu mōju kiirguse neeldumisele. Tulemusi võrreldakse teiste uurijate resultaatidega. Eraldi uuritakse, kuidas arvestada kiirgusnomogrammi konstrueerimisel läbilaskefunktsiooni sõltuvust temperatuurist. Näidatakse, et küllalt täpsed resultaadid saab, kui määrata atmosfääri integ raalne läbilaskefunktsioon mingi keskmise temperatuuri jaoks.

Eesti NSV Teaduste Akadeemia

Füüsika ja Astronoomia Instituut
Saabus toimetusse 10. V1 1961

\section{A NEW RADIATION CHART}

\section{Helgi Niilisk}

\section{Summary}

The aim of the present paper is to improve the graphic methods for computing the infra-red fluxes in the atmosphere. The data on the latest experimental and theoretical investigations have been used. The integral transmission function of the atmosphere has been determined, with the consideration of the influence of water vapour, carbon dioxide and ozone. A new, complete radiation chart has been constructed, using the given transmission function. The mean values of the infra-red radiation fluxes for the different zones of the Earth at a height of $0 ; 3$ and $8 \mathrm{~km}$ are calculated on the basis of the new radiation chart. The changes in pressure dependent on height have been taken into account. The results are compared with those of several authors. The influence of the temperature dependence for the transmission function on the results of the computation of infra-red fluxes has been considered. It has been shown that accurate results are obtained at the determining the integral transmission function for a mean temperature.

Academy of Sciences of the Estonian S.S.R., Institute of Physics and Astronomy
Received June 10th, 1961 